\title{
Does an Early Implantation of Extracorporeal Membrane Oxygenator in High-Risk Emergency Open Heart Surgery Patients Improve the Outcome in Comparison with Elective Patients? A Prospective Cohort Comparative Study
}

\author{
Ahmed Abdeljawad, MD, ${ }^{1,2}$ Yasser Shaban Mubarak, MD ${ }^{3,4}$ \\ ${ }^{1}$ Department of Cardiothoracic Surgery, Cairo University, Cairo, Egypt; \\ ${ }^{2}$ Clinic of Cardiac Surgery, Barmherzigen Brüder Krankenhaus Trier, Trier, Germany; \\ ${ }^{3}$ Department of Cardiothoracic Surgery, Minia University, Minia, Egypt; \\ ${ }^{4}$ Madinah Cardiac Center, King Fahd Hospital, KSA
}

\section{ABSTRACT}

Background: Extracorporeal membrane oxygenator $(\mathrm{ECMO})$ has been implemented in refractory postcardiotomy cardiogenic shock (PCCS) patients to maintain excellent oxygenation and hemodynamic support. The aim of this study is to compare the results of early ECMO implantation to treat refractory PCCS in emergency versus elective patients who developed univentricular or biventricular pump failure.

Patients and methods: Between January 2019 and June 2021, 35 patients received ECMO after refractory PCCS. Patients have been categorized into two groups: Group A contains 18 patients who were urgently operated on and Group $\mathrm{B}$, which includes 17 patients who were electively operated on. ECMO was implanted through central cannulation (right atrium and ascending aorta), or through peripheral cannulation (femoral vessels or through axillary artery).

Results: There was no statistically significant difference between the two ECMO groups in the preoperative patient's characteristics, complication rate, duration of mechanical ventilation, post-ECMO weaning hospital stay, duration of ICU stay, in-hospital mortality, and number of patients discharged from the hospital or in 1-year survival on follow up.

Conclusion: Early use of ECMO in high-risk emergency cardiac surgery should be taken into consideration when possible, without hesitance. Emergency and elective patients benefit equally from ECMO implantation and show comparable complication rates.

\section{INTRODUCTION}

Refractory PCCS after cardiac surgery ranges from $0.5-1.5 \%$ [Smedira 2001]. The first use of extracorporeal

Received Novembe 20, 2021; accepted December 16, 2021.

Correspondence: Abmed Abdeljawad, Telephone +491638144990 ,

+201221152534 (e-mail: abmed.gawad@kasralainy.edu.eg). membrane oxygenator (ECMO) as a treatment option for PCCS was introduced by Hill and colleagues, providing a prolonged temporary cardiopulmonary support [Hill 1972]. Severe PCCS as a sequel for refractory myocardial dysfunction is a serious complication with $100 \%$ mortality; however, the use of ECMO in that group of patients might provide hemodynamic support as a bridge to allow recovery from reversible myocardial dysfunction [Smedira 2001]. The early utilization of ECMO demonstrated better results compared with late implementation [Smith 2001]. The use of ECMO is associated with a high morbidity, including bleeding, renal failure, infection and neurological insult; however, the benefits that can be gained from ECMO implementation, regarding the cardiopulmonary support to allow recovery from the refractory myocardial injury, could outweigh the perioperative morbidity [Muehrcke 1996].

\section{PATIENTS AND METHODS}

Between January 2019 and June 2021, 35 patients with refractory PCCS were placed on Arterio-Venous (A-V) ECMO using heparin-coated circuits that consist of centrifugal pump console either Biomedicus (Medtronic) or JostraMaquet (Jostra Medizintechnik, Germany). These centrifugal pumps propel blood through a hollow-fiber membrane oxygenator (Affinity NTTM; Medtronic or Medos Medizintechnik AG, Stolberg, Germany). (Figure 1) (Figure 2) Our indication to implant ECMO was for patients who could not be weaned from cardiopulmonary bypass $(\mathrm{CPB})$ because of refractory PCCS. Our criteria to define PCCS include failure to build up a systolic pressure above $80 \mathrm{mmHg}$, persistent metabolic acidosis in serial blood gas analysis samples (e.g. $\mathrm{pH}<7.3$, lactate level $>3 \mathrm{mmol} / \mathrm{L}$ ) in spite of using maximal support by various means including catecholamines, phosphodiesterase inhibitors, and the utilization of IABP.

ECMO was implanted while the patient was fully heparinized with activated clotting time (ACT) $>350$ seconds. Once ECMO flow was established heparin was antagonized, however the aim was to keep ACT $\geq 180$ seconds and to keep partial thromboplastin time (PTT) between 50-60 seconds. The aim of ECMO implantation was reduction of inotropic 


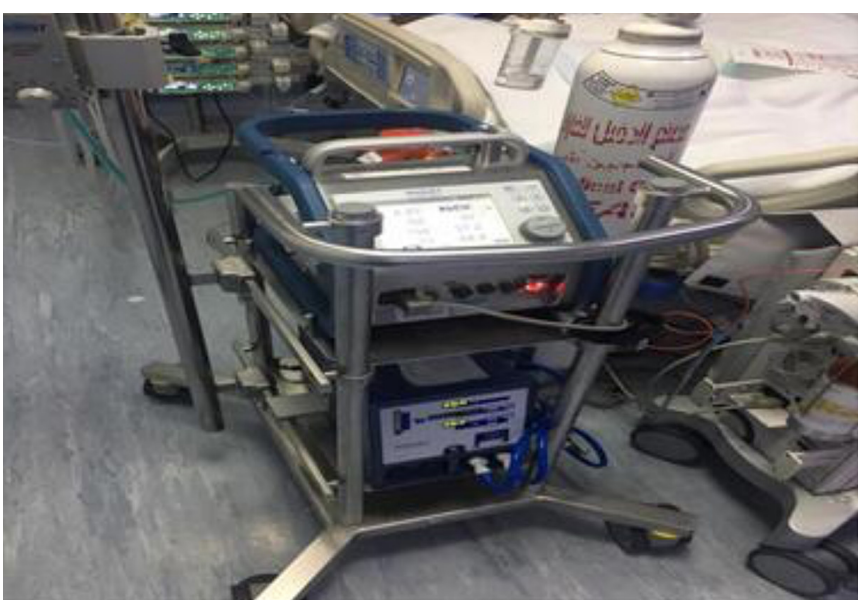

Figure 1. ECMO machine

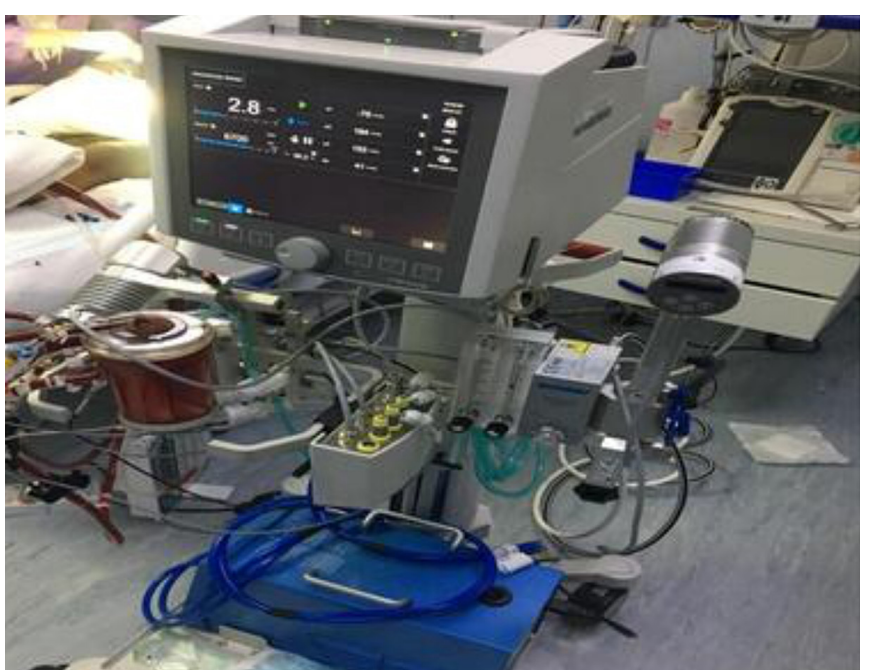

Figure 2. ECMO machine

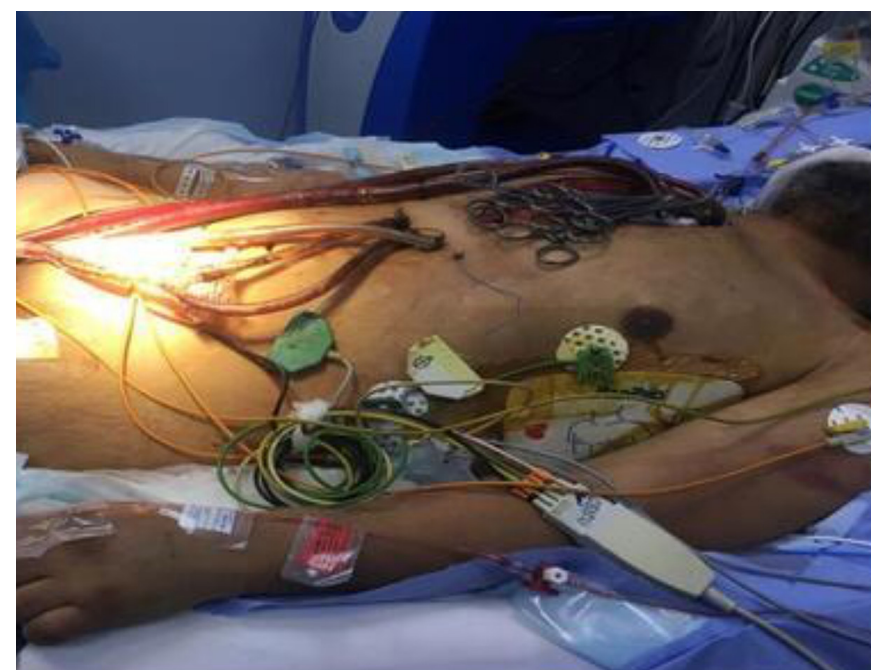

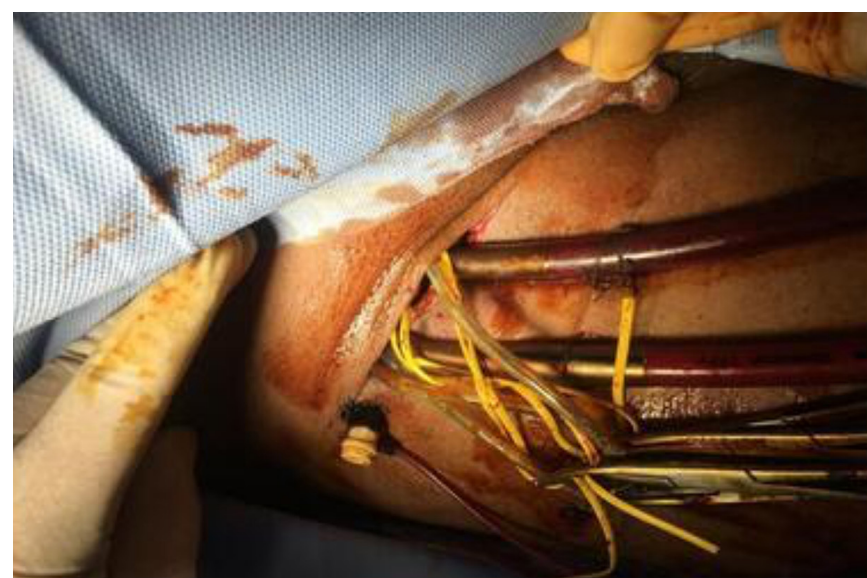

Figure 4. Peripheral cannulation ECMO (Femoral access)

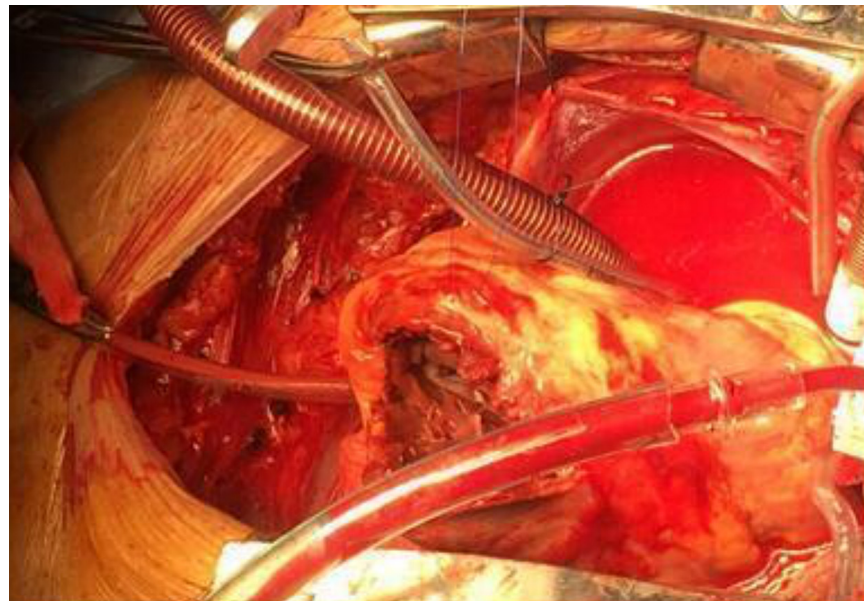

Figure 5. Intra-operative VSR

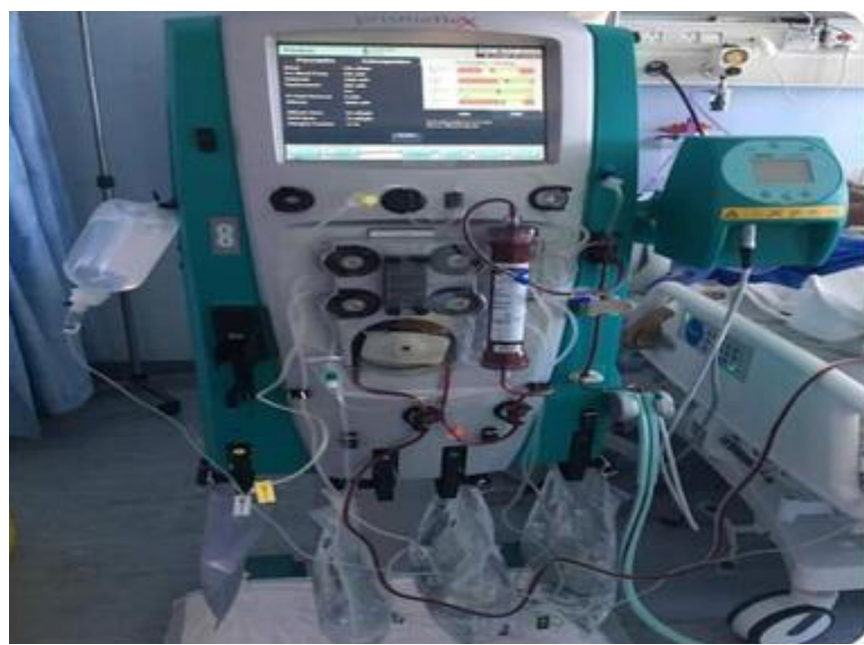

Figure 6. Continuous renal replacement therapy

Figure 3. Central cannulation ECMO with cell-saver connected chest drains 
support and optimizing blood oxygen saturation. The membrane oxygenator was examined periodically through the whole day for early detection of blood clots or thrombus formation, which was reflected in the form of sudden elevation in the perfusion pressure with continuous alarming. To monitor the flow across the ECMO, a Doppler flow probe was placed and attached to the arterial side of the circuit.

Arterial cannulation was central in all group A patients and in $70 \%$ of group B patients (12 patients), where the arterial cannula was inserted in the aorta and the venous cannula was inserted in the right atrial appendage. (Figure 3)

Among the $30 \%$ patients of the group B (5 patients) in whom peripheral cannulation was performed, arterial cannulation access was implemented through the femoral artery in $80 \%$ of patients ( 4 patients) and through the axillary artery in $20 \%$ of patients (one patient). (Figure 4 )

Central cannulation (ascending aorta and right atrium) was carried out in 30 patients $(86 \%)$, peripheral cannulation (common femoral artery and femoral vein) was carried out in 4 patients $(11 \%)$, and axillary artery and femoral vein in only one patient $(3 \%)$. We divided the patients in our study into two groups: group A contained 18 patients who were operated upon on an emergency basis. This included 10 patients of ischemic heart disease (IHD) with NSTEMI, 3 patients of IHD with severe ischemic mitral regurgitation (IMR), 2 patients of IHD complicated by ventricular septal rupture (VSR) (Figure 5), 1 patient of redo infective endocarditis affecting biological aortic valve prosthesis, 2 patients of severe MR (one due to traumatic papillary muscle rupture and the other - a female patient who developed a pulmonary edema on top of severe mitral stenosis refractory to medical therapy). (Figure 5) ECMO implantation in group A took place intraoperatively with central cannulation in all patients. Group B included 17 patients, who were operated upon on an elective basis. This included 9 patients of IHD with impaired pump function, 2 patients of IHD with severe ischemic mitral regurgitation (IMR), 3 patients with AVD, and 3 patients with mitral valve disease. In group B, ECMO was implanted in 12 patients intraoperatively with central cannulation after failure to wean from CPB and after optimizing the inotropic support; in 5 patients, the ECMO with peripheral cannulation was established after the patient was transported to ICU within less than $24 \mathrm{hr}$ and after confirming the diagnosis of PCCS.

Our weaning strategy was based on establishing an ECMO flow for at least $72 \mathrm{hr}$ before starting the weaning process. Weaning was based on the degree of improvement of hemodynamic and metabolic signs. Transesophageal echo (TEE) was performed on a daily basis and especially before the start of weaning. ECMO weaning was discontinued when signs of malperfusion or metabolic acidosis appeared during the weaning process. At this point, weaning was discontinued and ECMO flow was readjusted on the pre-weaning ECMO flow level and kept for at least $36-48 \mathrm{hr}$ before repeating the weaning process.

Statistical analysis: Continuous variables are expressed as mean $\pm \mathrm{SD}$ and were evaluated by using Student t-test or Kruskal-Wallis test. Categorical variables were compared

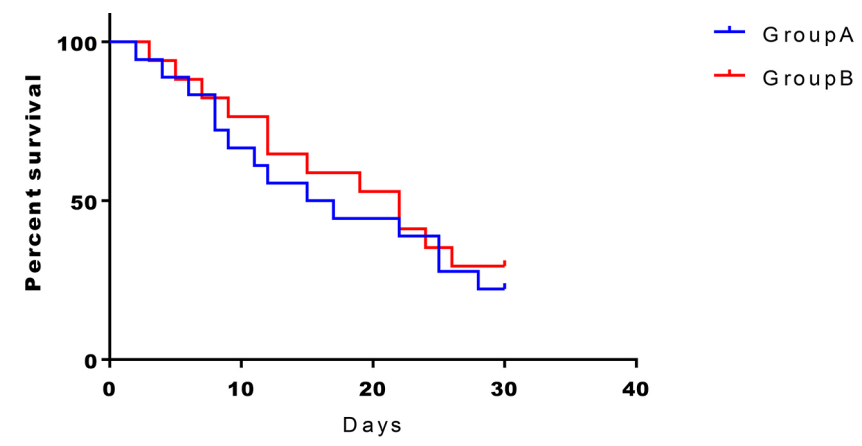

Figure 7. Kaplan Meier survival curve of both groups of patients showing in-hospital 30 days survival.

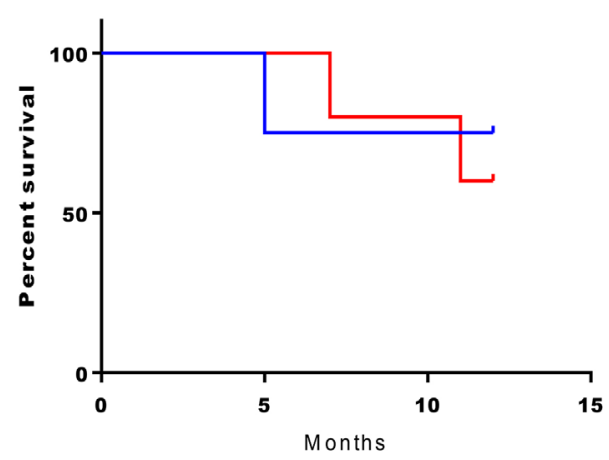

Figure 8. Kaplan Meier survival curve showing 1-year survival of both groups

with Pearson's chi-squared test. Long-term survival (one year in our study) was calculated according to Kaplan Meier method, which was used to analyze 'time-to-event' data.

\section{RESULTS}

Table 1 shows the underlying cardiac disease and the corresponding surgical cardiac procedure of the patients who received ECMO implantation in the two groups. (Table 1)

Table 2 shows the preoperative patient's characteristics. (Table 2) There was no statistically significant difference in the preoperative patient's characteristics between the two groups.

Table 3 shows the complications that occurred among patients, who had undergone ECMO implantation. (Table 3) There was a considerable postoperative complication among patients who received ECMO implantation in both groups. There was no statistically significant difference in the complication rate between emergency and elective surgical cases. Massive blood loss that necessitated re-exploration for bleeding, together with high incidence of infection (43\%) and acute renal failure (74\%), significantly impaired in-hospital survival. Re-exploration was done by repeated dressing, surgical suture control, and hemostatic material (surgicel, gelfoam sponge, etc.). Drains had been connected to cell-saver and lost blood was reinfused. Deficient coagulation factors were replaced 
Table 1. Operative procedures in both groups

\begin{tabular}{lcc}
\hline Operative procedure & Group A (Emergency) $(N=18)$ & Group B (Elective) $(N=17)$ \\
\hline CABG & 10 patients with acute MI & 9 patients \\
CABG + MVR for IMR & 3 patients & 2 patients \\
CABG + VSR & 2 patients & 0 patients \\
AVR & 1 patient & 3 patients \\
MVR & 2 patients & 3 patients
\end{tabular}

Table 2. Preoperative patient characteristics (total number including emergency and elective)

\begin{tabular}{|c|c|c|c|c|c|c|}
\hline No. & 35 & 19 & 5 & 2 & 5 & 4 \\
\hline Male gender & 20 (57\%), Group A 9 (45\%), Group B 11 (55\%) & $12(34 \%)$ & $3(8 \%)$ & $2(6 \%)$ & $2(6 \%)$ & $1(3 \%)$ \\
\hline Female gender & 15 (43\%), Group A 9 (60\%), Group B 6 (40\%) & $6(17 \%)$ & $2(6 \%)$ & $1(3 \%)$ & $4(11 \%)$ & $2(6 \%)$ \\
\hline HTN & 20 (57\%), Group A 9 (45\%), Group B 11 (55\%) & $15(43 \%)$ & $2(6 \%)$ & $2(6 \%)$ & $1(3 \%)$ & 0 \\
\hline LVEF \% & $25.6 \pm 4.3$ & $25 \pm 7.2$ & $23 \pm 4.5$ & $21 \pm 3.1$ & $28 \pm 2.2$ & $31 \pm 4.8$ \\
\hline BMI & $25.2 \pm 2.5$ & $29 \pm 2.5$ & $27 \pm 1.7$ & $25 \pm 3.7$ & $22 \pm 1.8$ & $23 \pm 3.1$ \\
\hline
\end{tabular}

Table 3. Postoperative associated complications

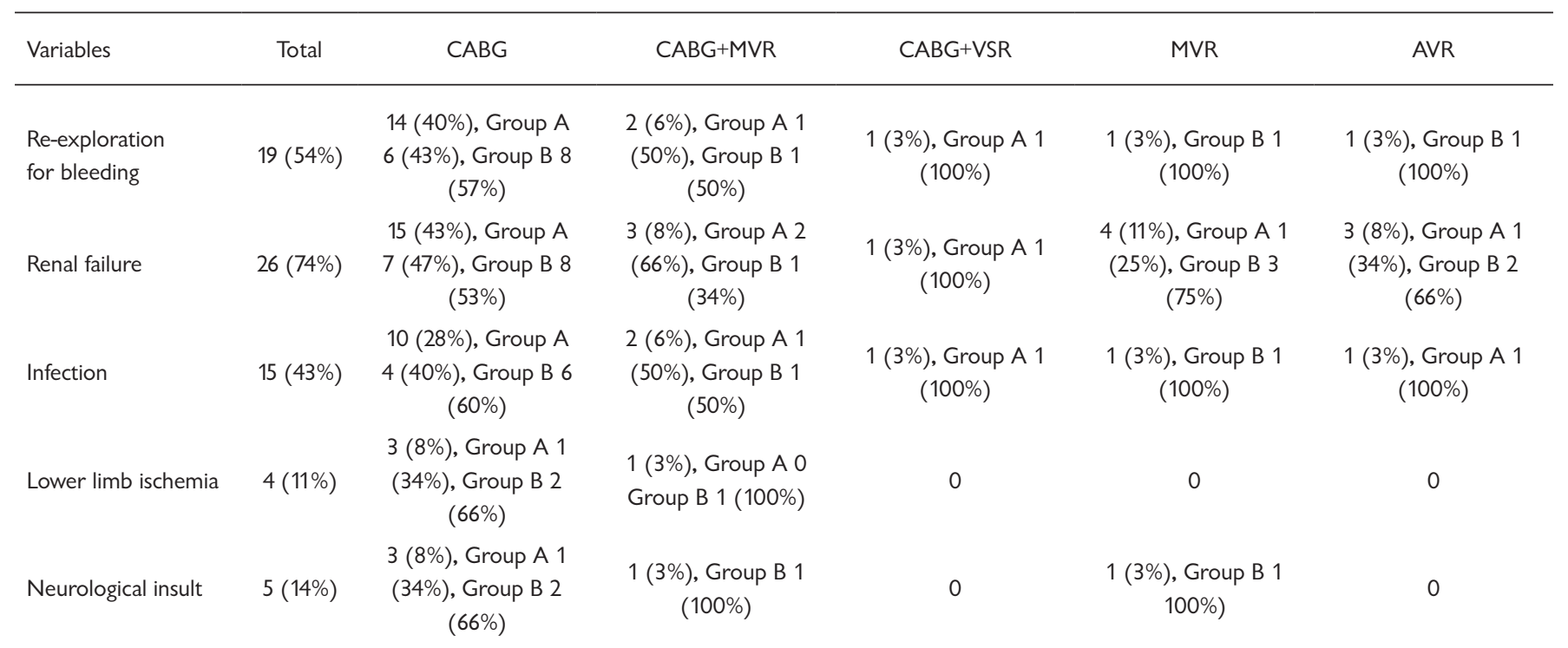


Table 4. Intraoperative data

\begin{tabular}{|c|c|c|c|c|c|c|}
\hline & 25 (71\%), Group A & & & & & \\
\hline Use of IABP & & $132.5 \pm 62.5$, Group & $157 \pm 37.5$, Group A & & $42.5 \pm 20.5$, Group & $26 \pm 14$, Group A \\
\hline CPB time (min) & $105.6 \pm 31.5$ & $\begin{array}{c}\text { A } 135 \pm 65 \text {, Group B } \\
130 \pm 60\end{array}$ & $\begin{array}{c}160 \pm 35 \text {, Group B } \\
155 \pm 40\end{array}$ & Group A $170 \pm 23$ & $\begin{array}{c}\text { A } 40 \pm 23 \text {, Group B } \\
45 \pm 18\end{array}$ & $\begin{array}{c}28 \pm 12 \text {, Group B } \\
24 \pm 16\end{array}$ \\
\hline
\end{tabular}

Table 5. Comparison of ECMO results in both groups

\begin{tabular}{lcc}
\hline Variables & Group A & Group B \\
\hline ECMO duration (days) & $6.8 \pm 3.2$ & $7.3 \pm 2.1$ \\
Weaning success & $9(25 \%)$ & $8(23 \%)$ \\
Duration of mechanical ventilation (days) & $10 \pm 4$ & $9 \pm 3$ \\
ICU stay (days) & $24 \pm 9$ & $22 \pm 8$ \\
Post ECMO weaning hospital stay (days) & $32 \pm 5$ & $27 \pm 3$ \\
In hospital mortality ( $N=26$ ) & $14(40 \%)$ & $12(34 \%)$ \\
30 days survival & 4 (11\%), 4 patients emergency CABG & $5(14 \%)$, CABG (3), CABG and MVR (1), AVR (1) \\
1-year survival on follow up & 3 out of 4 (75\%) & 3 out of 5 (60\%)
\end{tabular}

by blood products. The infections we faced during our study included chest infection, wound infection, and sever septicemia. The patients who developed acute kidney failure needed CRRT many times until recovery. (Figure 6)

Table 4 shows the intraoperative data of both groups. (Table 4) IAPB was implanted in 25 patients of both groups ( $71 \%)$, but it was more used in CABG. IABP intraoperatively was inserted in 20 patients (10 in group A and 10 in group B). IABP postoperatively was inserted in the ICU in 5 patients ( 3 in group A and 2 in group B).

The mean $\mathrm{CPB}$ time in both groups was 105.6 \pm 31.5 minute, which was significantly longer in patients who had undergone CABG with MVR $(157 \pm 37.5$ minutes $)$ and in the two patients who had undergone CABG with VSR closure (170 \pm 23 minutes).

Table 5 shows the outcome of ECMO implantation in the two groups. (Table 5) The mean duration of ECMO support was $6.8 \pm 3.2$ days in group A and $7.3 \pm 2.1$ days in group B, which was found to be statistically insignificant.

Successful weaning from ECMO has been reported in 9 patients from group A (50\%) and 8 patients from group B (47\%), without a statistically significant difference $(P=0.29)$. Five patients from group $\mathrm{A}$ and 3 patients from group $\mathrm{B}$ who were successfully weaned from ECMO died during their hospital stay, due to septicemia, persistent renal failure and congestive heart failure.

There was no statistically significant difference between the two groups, regarding the duration of mechanical ventilation $(P=0.84)$, duration of ICU stay $(P=0.19)$, post ECMO weaning hospital stay (days) $(P=0.92)$, in-hospital mortality
$(P=0.49)$, the number of patients who were discharged from the hospital $(P=0.38)$, and one-year survival on follow up $(P=$ 0.70). Kaplan Meier curves show the in-hospital 30 days and 1 year survival, respectively. (Figure 7) (Figure 8)

It is worthwhile to mention that at the beginning of our research, we postulated there will be a statistically significant difference between emergency operated and electively operated patients, who would receive ECMO implantation, regarding in-hospital mortality and in the number of patients who would be discharged from the hospital. However, at the end of our study, we did not record a significant difference between two groups.

\section{DISCUSSION}

PCCS remains the most serious major complication in cardiac surgery, especially in patients who preoperatively demonstrate heart failure symptoms. Persistent myocardial dysfunction has been demonstrated in $2-5 \%$ of patients, during weaning from $\mathrm{CPB}$, despite the use of inotropic drugs and IABP. The use of ECMO as a sort of extracorporeal life support (ECLS) in patients, who develop PCCS, has introduced new unique complications, including high bleeding and re-exploration, renal failure, and increased infection rate. Despite these complications, ECMO remains an excellent means of life support with a variety of advantages, including the ease of rapid deployment in emergency situations, improvement of tissue oxygenation, and providing a biventricular support [Kawahito 1994]. 
Muehrcke and associates reported a survival rate of $30.4 \%$ [Muehrcke 1996]. Kawahito et al. reported a survival rate of $39 \%$ in 13 patients with PCCS [Kawahito 1994]. In our study, we report a survival rate of $26 \%$; however, the better results they reported can be attributed to the small number of patients in their study, which is smaller than $50 \%$ the number of our study patients.

Our in-hospital mortality was $74 \%$, which is comparable to in-hospital mortality reported by Bakhtiary et al., who reported $71 \%$ in-hospital mortality rate [Bakhtiary 2008]. Surprisingly, we did not notice a statistically significant difference, regarding in-hospital mortality between patients who were urgently operated on (77\%) and those who were electively operated on $(70.5 \%)$, taking in to consideration their preoperative high-risk score. We did not record a significant increase in in-hospital mortality as a sequel of delayed ECMO implantation (within <24 hours) as we did not report a significant rise in in-hospital mortality in emergency patients who intraoperatively received ECMO.

Mean ECMO duration in our patient groups was 7.0 \pm 2.6 days, which is comparable to that reported by Bakhtiary et al. [Bakhtiary 2008]. Our ECMO duration was longer than that reported in other studies [Hill 1972; Doll 2003; Pagani 1999].

Our postulation at the start of our study of unsatisfactory results in patients who had been urgently operated and in whom the ECMO implantation took place immediately after failure to wean from CPB after reaching optimum pharmacological support and IABP insertion was not proven at the end of our study. We found that early use of ECMO did not add any significant increase in mortality or procedure-associated morbidity, including rate of bleeding, re-exploration, infection, or renal failure. One-year survival follow up in our study was short; however, we noticed a short-term survival rate among patients who had been discharged from the hospital (75\% of the discharged patients in group A and $60 \%$ of the discharged patients in group B).

ECMO implantation was not free of some unique significant complications that added to morbidity in these patients. Excessive bleeding that necessitated re-exploration was reported in almost $50 \%$ of both groups. Golding and colleagues reported a re-exploration rate of $87 \%$ among 91 patients; this is more than double the number of patients included in our study [Golding 1992]. Rastan and associates reported a re-exploration rate of 58\% [Rastan 2010].

We reported a lower limb ischemia that necessitated removal of cannula and fasciotomy in $6 \%$ of our patients; this was found to be less than that reported by Rastan and associates who reported leg ischemia in 19.9\% [Rastan 2010].

We reported a renal failure that necessitated hemodialysis in $74 \%$ of our patients; this is comparable to that reported by Bakhtiary et al. (87\%) [Bakhtiary 2008], and that reported by Rastan et al. (65\%). This confirms the results shown in a systemic review included in 46 studies in patients who received ECMO, where occurrence rate was reported to be $53 \%$ [Rastan 2010].

We reported neurological events in 14\% of our patients. Smedira and colleagues reported 33\% among 202 patients [Smedira 2001]. In autopsy studies performed by Rastan and colleagues, $51.9 \%$ of all pathological cerebral events in patients undergoing ECMO were found to be clinically undetectable [Rastan 2006].

In our study, 10 patients received urgent CABG followed by intraoperative ECMO implantation. Hospital survival was $40 \%$ and 1 -year survival was $75 \%$. Our hospital survival results were not superior to those reported by Wagner and associates, who reported hospital survival of $68.2 \%$ and 1-year survival of 59.1\% [Wagner 2019].

Our findings of improved survival of emergency patients, which is comparable to the survival of high-risk elective patients, could be attributed to the fact that early intraoperative ECMO implantation in emergency patients or shortly in the postoperative course had aided in heart muscle regeneration via temporary circulatory support. This could be demonstrated and proven by the obvious improvement in the pump function at the ICU, as reflected in the improvement of EF values in serial TEE examinations.

Furthermore, owing to the protective role provided via ECMO implantation at an early stage, we could reduce inotropic supports to the minimal level that could maintain an optimal organ perfusion. Early ECMO implantation protected these patients from exposure to a large toxic catecholamine dose.

\section{CONCLUSION}

Early use of ECMO in high-risk emergency cardiac surgery should be considered, when possible, without hesitance. Emergency and elective patients benefit equally from ECMO implantation and show comparable complication rates.

\section{REFERENCES}

Bakhtiary F, Keller H, Dogan S, Dzemali O, Oezaslan F, Meininger D, et al. 2008. Veno-arterial extracorporeal membrane oxygenation for treatment of cardiogenic shock : clinical experiences in 45 adult patients. J Thorac Cardiovasc Surg. 135:382-88.

Doll N, Fabricius A, Borger MA, Bucerius J, Doll S, Kramer K, et al. 2003. Temporary extracorporeal membrane oxygenation in patients with refractory postoperative cardiogenic shock - a single center experience. J Card Surg. 18:512-18.

Golding LA, Crouch RD, Stewart RW, Novoa R, Lytle BW, McCarthy PM, et al. 1992. Postcardiotomy centrifugal mechanical ventricular support. Ann Thorac Surg. 54:1059-64.

Hill D, O`Brien TG, Murray JJ, Dontigny L, Bramson ML, Osborn JJ, et al. 1972. Prolonged extra-corporeal oxygenation of acute post traumatic respiratory failure (shock lung syndrome).

N Engl J Med. 286:629-34.

Kawahito K, Ino T, Adachi H, Mizuhara A, Yamaguci A. 1994. Heparin coated percutaneous cardiopulmonary support for the treatment of circulatory collapse after cardiac surgery. ASAIO Trans. 40:972-76.

Morris AH. 2006. Extracorporeal support and patient outcome: credible causality remains elusive. Critical Care Med. 34:1551-52.

Muehrcke DD, McCarthy PM, Stewart RW, Foster RC, Ogella DA, 
Borsh JA, et al. 1996. Extracorporeal membrane oxygenation for postcardiotomy cardiogenic shock. Ann Thorac Surg. 61: 684-91.

Pagani FD, Lynch W, Swaniker F, Dyke DB, Bartlett R, Koelling T, et al. 1999. Extracorporeal life support to left ventricular assist device Bridge to heart transplant: a strategy to optimize survival and resource utilization. Circulation. 2:206-10.

Rastan AJ, Dege A, Mohr M, Doll N, Falk V, Walther T, et al. 2010. Early and late outcomes of 517 consecutive adult patients treated with extracorporeal membrane oxygenation for refractory postcardiotomy cardiogenic shock. J Thorac Cardiovasc Surg. 139:302-11.

Rastan AL, Lachmann N, Walther T, Doll N, Gradistance T, Gummert JF, et al. 2006. Autopsy findings in patients on postcardiotomy extracorporeal membrane oxygenation (ECMO). Int J Artif Organs. 29: 1121-31.
Smedira NG, Moazami N, Golding CM, McCarthy PM, AppersonHansen C, Blackstone EH, et al. 2001. Clinical experience with 202 adults receiving extracorporeal membrane oxygenation for cardiac failure ; survival at five years. J Thorac Cardiovasc Surg. 122:92-102.

Smith C, Bellomo R, Raman JS, Matalanis G, Rosalion A, Buckmaster J, et al. 2001. An extracorporeal membrane oxygenation based approach to cardiogenic shock in an older population. Ann Thorac Surg. 71:1421-27.

Thiagarajan RR, Bratton SL. 2006. Extracorporeal membrane oxygenation for cardiac arrest: when to use it, and what are the outcomes? Critical Care Med. 34:1285-86.

Wagner J, Tiller C, Dietl M, Ulmer H, Brenner C, Stastny L, et al. 2019. Extracorporeal life support in myocardial infarction-induced cardiogenic shock: weaning success. Ann Thorac Surg. 108:1383-90. 